\title{
Utilización de fuentes de energías renovables en los puertos brasileños
}

\author{
Dayla Karolina Fossile \\ dfossile@bol.com.br \\ Pontifícia Universidades Católica do \\ Paraná - PUCPR. Curitiba. Paraná. Brasil. \\ Segio Eduardo Gouvea da Costa \\ s.gouvea@pucpr.com \\ Pontifícia Universidades Católica do \\ Paraná - PUCPR. Curitiba. Paraná. Brasil. \\ Edson Pinheiro de Lima \\ e.pinheiro@pucpr.com \\ Pontifícia Universidades Católica do \\ Paraná - PUCPR. Curitiba. Paraná. Brasil.
}

\begin{abstract}
RESUMEN
Esta investigación presenta un modelo de decisión para resolver un problema de selección, identificando qué fuente de energía renovable es la más viable para invertir en los puertos brasileños, considerando los aspectos de la gestión ambiental, social y económica, utilizando el Método PROMETHEE (Preference Ranking Organization Method for Enrichment Evaluations). Método de organización para evaluaciones de enriquecimiento) mediante la aplicación del software Visual PROMETHEE 1.4. Sin embargo, esta investigación evaluó tres fuentes de energía limpia (eólica, fotovoltaica y undimotriz), definiendo veinte criterios, basados en el conocimiento existente en investigación científica, instrucciones normativas, legislación, datos de proyectos ya instalados o en estudio. Observando que estos criterios fueron evaluados por expertos de la Empresa de Investigación Energética (EPE, en portugués brasileño) en una escala liker de cinco puntos. Sin embargo, la evaluación realizada en este estudio revela que la energía fotovoltaica es la fuente de energía renovable más viable para que los puertos brasileños inviertan.
\end{abstract}

PALABRAS CLAVE: Energia renovable, multicriterios, puertos, sostenibilidad, PROMETHEE. 


\section{INTRODUÇÃO}

Desde la década de los 50 muchos cambios han ocurrido, especialmente en los procesos productivos empresariales, poniendo las fuentes de energía como una nueva perspectiva para el desarrollo de compañías (GLENDENNING, 1976). Segundo Glendenning (1976), en 1974, la División de Investigación Central de Generación de Energía Eléctrica empezó la evaluación de la utilización de fuentes de energía renovable para las compañías. En 1975, se debatían los problemas de la producción energética y sus impactos al medio ambiente. Además, había discusiones sobre la crisis energética y su papel en los futuros procesos empresariales (ECKERT et al., 1976).

De acuerdo con Çelikle y Askan (2016), aproximadamente 74\% de la demanda de la energía mundial es atendida por la generación de energía no renovable, $6 \%$ es atendida por energía nuclear, mientras que $20 \%$ de la demanda es atendida por energía limpia. La energía renovable es considerada relativamente joven, pues fue impulsada en 1997 por la descarburación del sector energético (PASKA; SURMA, 2014).

Es importante destacar que, para superar el efecto del calentamiento global, es necesario reducir la emisión de $\mathrm{CO} 2$. Así, los países deben establecer una política para estimular que las empresas puedan utilizar fuentes de energía renovable (LIRN; WU; CHEN, 2013).

Cuando se habla de los puertos a nivel mundial, muchos utilizan fuentes de energía limpia, como, por ejemplo: el Puerto de Róterdam (Holanda), el Puerto de Génova (Italia), el Puerto de Hartlepool en el Reino Unido, el Puerto de Kitakyushu en Japón, el Puerto de Los Ángeles en los Estados Unidos, así como los Puertos de Tokio y San Diego (ACCIARO; GHIARA; CUSANO, 2014; RAMOS et al. 2014; BALDENEGRO, 2013).

Los puertos europeos en mayoría utilizan fuentes de energía limpia, con el principal objetivo de reducir las emisiones de $\mathrm{CO} 2$, reducir costos y ampliar la eficiencia de la sostenibilidad portuaria (MOUTINHO; ROBAINA, 2016). Las fuentes de energía renovable más utilizadas son: eólica, fotovoltaica y undimotriz (FOSSILE; GOUVEA DA COSTA; PINHEIRO DE LIMA, 2016).

Con esto, es posible percibir que se viene discutiendo por algún tiempo la utilización de fuentes de energía renovable. Aunque, en el sector de puertos brasileño, se puede observar que, en el principio de los 90, hubo una reformulación de la legislación portuaria. Pero, la Ley 8.630/93 no destacaba la utilización de la gestión de sostenibilidad, mucho menos la utilización de la gestión de eficiencia energética.

Sin embargo, la Autoridad de Transportes Acuaviarios (ANTAQ, en portugués brasileño), basada en la nueva legislación portuaria brasileña, la Ley 12.815/2013, cree que los puertos deben implementar la gestión ambiental, a través de la utilización del Índice de Desempeño Ambiental (IDA). El índice aborda la utilización de fuentes renovables, ya que los puertos son considerados un segmento extremadamente contaminante (ROOS; KLEIMANN NETO, 2015).

Así, es posible percibir como está esencial utilizar fuentes de energía renovable en las empresas portuarias brasileñas. El problema de investigación puede ser resumido en la siguiente pregunta: ¿Cuál es la fuente de energía 
renovable (eólica, fotovoltaica o undimotriz) más viable para ser utilizada en los puertos brasileños, considerando la gestión de sostenibilidad?

El objetivo de la investigación es realizar un análisis multicriterio con el propósito de evaluar la fuente de energía renovable más viable para ser utilizada en los puertos brasileños, considerando los aspectos de la gestión de sostenibilidad.

Este estudio se divide en cuatro secciones con la introducción. La segunda sección trae los procedimientos tecnológicos de la investigación. La tercera sección habla del análisis de los resultados y, finalmente, se presenta la conclusión del estudio.

\section{PROCEDIMIENTOS METODOLÓGICOS}

El enfoque metodológico utilizado en la investigación es un método multicriterio; son utilizadas técnicas que van evaluar varios criterios que son importantes para la toma de decisiones en un proceso o en un proyecto. En este estudio, se propone un procedimiento para resolver un problema de decisión multicriterio, en tres fases distintas.

La primera fase es llamada preliminar, empieza con la contextualización del problema para después caracterizar el responsable por la toma de decisiones y otros actores y, por fin, son establecidos las alternativas y criterios.

La segunda fase, llamada de modelaje de preferencias y selección del método multicritério. La última fase, llamada de finalización, empieza con el análisis de los resultados y, después, con el análisis de sensibilidad y de robustez del modelo creado. Por fin, son identificadas las oportunidades y las recomendaciones para la mejora continua de su desempeño (de ALMEIDA, et al. 2015).

El método PROMETHEE, utilizado en este estudio, no ofrece orientaciones específicas para determinar los pesos de los criterios. Además, la definición de la importancia exacta de los pesos de los criterios es una tarea difícil, puesto que las situaciones imprecisas están presentes en la vida real (MORAES et al. 2015; MACHARIS et al. 2004).

De acuerdo con Brans y Vincke (1985), es importante notar que, a través de la utilización de este método, hay condiciones para hacer comparaciones y clasificar varias alternativas, de naturaleza cualitativa o cuantitativa.

\section{ANALISIS DE LOS RESULTADOS}

Esta sección se refiere a la aplicación numérica del sistema de apoyo a la toma de decisiones PROMETHEE, con el propósito de seleccionar la fuente de energía renovable más adecuada para la utilización de los puertos brasileños, tomando en cuenta los aspectos de la sostenibilidad.

Según el procedimiento para resolver el problema de decisión multicriterio, es necesario definir el decisor y otros actores del proceso decisorio. Para este estudio, los decisores son los gestores y las autoridades portuarios. En este proceso están involucrados otros actores como: analistas de los departamentos 
medioambientales, sociales y económicos, así como, expertos en fuentes de energía renovable.

Para la definición del conjunto de criterios, informaciones presentadas en la literatura fueron utilizadas como base juntamente con leyes, resoluciones, emprendimientos ya implementados o en estudio, informaciones de los decisores y expertos. Un total de 20 criterios de acuerdo con la mensurabilidad, operabilidad, comprensibilidad, legitimidad y homogeneidad (ENSSLIN; ENSSLIN, 2009; ENSSLIN; MONTIBELLER; NORONHA, 2001). La Tabla I presenta una descripción breve de los criterios.

Tabla I: Descripción de los criterios

\begin{tabular}{|c|c|}
\hline Criterio & Descripción \\
\hline CA1 Terrestre & $\begin{array}{l}\text { Este es un criterio ambiental (CA) para valorar la necesidad de extensiones terrestres y supresión } \\
\text { de vegetación }\end{array}$ \\
\hline CA2 Marina & $\begin{array}{l}\text { Este es un criterio ambiental (CA) para valorar la necesidad de extensiones marinas y supresión } \\
\text { de vegetación fluvio-marina }\end{array}$ \\
\hline CA3 Contaminación & $\begin{array}{l}\text { Este es un criterio ambiental (CA) para valorar la posibilidad de contaminación de las aguas } \\
\text { marítimas }\end{array}$ \\
\hline $\begin{array}{l}\text { CA4 Tóxicos y } \\
\text { contaminantes }\end{array}$ & $\begin{array}{l}\text { Este es un criterio ambiental (CA) para valorar la utilización de los productos tóxicos y } \\
\text { contaminantes en el proceso productivo utilizado en los emprendimientos de energía renovable }\end{array}$ \\
\hline CA5 Reciclaje & $\begin{array}{l}\text { Este es un criterio ambiental (CA) para valorar la necesidad constante de reciclaje de los } \\
\text { emprendimientos de energía renovable }\end{array}$ \\
\hline CA6 Burocracia & $\begin{array}{l}\text { Este es un criterio ambiental (CA) para valorar la burocracia de las expediciones de licencias } \\
\text { ambientales para cada emprendimientos de energía renovable }\end{array}$ \\
\hline CA7 Vías de acceso & $\begin{array}{l}\text { Este es un criterio (CA) para valorar la necesidad de construcción de vías de acceso para los } \\
\text { emprendimientos de energía renovable }\end{array}$ \\
\hline CS8 Desplazamiento & $\begin{array}{l}\text { Este es un criterio social (CS) para valorar la necesidad de desplazamiento de la comunidad local } \\
\text { con la instalación de las fuentes de energía renovable }\end{array}$ \\
\hline CS9 Ruidos & $\begin{array}{l}\text { Este es un criterio social (CS) para valorar el nivel de ruidos de los emprendimientos de energía } \\
\text { renovable }\end{array}$ \\
\hline $\begin{array}{l}\text { CS10 Navegación y } \\
\text { pesca }\end{array}$ & Este es un criterio social (CS) para valorar la limitación de la navegación y la pesca \\
\hline CS11 Deforestación & $\begin{array}{l}\text { Este es un criterio social (CS) para valorar la deforestación y la reducción de área verde para la } \\
\text { comunidad }\end{array}$ \\
\hline CS12 Salud y seguridad & $\begin{array}{l}\text { Este es un criterio social (CS) para valorar los riesgos y seguridad de los trabajadores de un } \\
\text { emprendimiento de energía renovable. }\end{array}$ \\
\hline CS13 Empleabilidad & $\begin{array}{l}\text { Este es un criterio social (CS) para valorar el nivel de empleabilidad de los emprendimientos de } \\
\text { energía renovable }\end{array}$ \\
\hline CE14 Eficiencia & $\begin{array}{l}\text { Este es un criterio económico (CE) para valorar la eficiencia de los emprendimientos de energía } \\
\text { renovable }\end{array}$ \\
\hline CE15 Inversiones & Este es un criterio económico (CE) para valorar la magnitud de la inversión inicial \\
\hline CE16 Incentivos fiscales & $\begin{array}{l}\text { Este es un criterio económico (CE) para valorar el volumen de incentivos fiscales ofrecidos por } \\
\text { el Gobierno de Brasil }\end{array}$ \\
\hline CE17 Costos & $\begin{array}{l}\text { Este es un criterio económico (CE) para valorar los costos de manutención anual de los } \\
\text { emprendimientos de energía renovable }\end{array}$ \\
\hline CE18 Financiación & Este es un criterio económico (CE) para valorar el plazo de la financiación ofrecida por lo BNDES \\
\hline $\begin{array}{l}\text { CE19 Tasa de } \\
\text { amortización }\end{array}$ & $\begin{array}{l}\text { Este es un criterio económico (CE) para valorar las tasas de depreciación de los equipos de las } \\
\text { fuentes de energía renovable según el MCPSE }\end{array}$ \\
\hline $\begin{array}{l}\text { CE20 Plazo para } \\
\text { instalación }\end{array}$ & $\begin{array}{l}\text { Este es un criterio económico (CE) para valorar el tiempo de instalación de los emprendimientos } \\
\text { de energía renovable }\end{array}$ \\
\hline
\end{tabular}
Fuente: Elaboración propia. 
En relación con la dimensión ambiental, no se consideró el criterio de emisiones de $\mathrm{CO} 2$, pues según informaciones de expertos, las fuentes de energía renovable dejan de emitir gases de efecto invernadero o emiten cantidades extremadamente insignificantes.

Para definir la escala de criterios descritos, una escala Liker de cinco puntos fue establecida ( 1 = completo desacuerdo; 2 = desacuerdo; 3 = indiferente; 4 = acuerdo; 5 = completo acuerdo).

Con las informaciones, una matriz de decisiones con el desempeño de cada alternativa para cada criterio fue creada. La Tabla II presenta la matriz.

Tabla II: Consecuencia de las fuentes de energía renovable

\begin{tabular}{|c|c|c|c|c|c|c|c|}
\hline \multicolumn{8}{|c|}{ Aspectos ambientales } \\
\hline Alternativas & CA1 & CA2 & CA3 & \begin{tabular}{l|r} 
& CA4 \\
\end{tabular} & CA5 & CA6 & CA7 \\
\hline Eólica & 4 & 1 & 1 & 4 & 3 & 4 & 4 \\
\hline Fotovoltaica & 2 & 1 & 1 & 5 & 4 & 3 & 1 \\
\hline Undimotriz & 4 & 5 & 4 & 4 & 3 & 5 & 4 \\
\hline \multicolumn{8}{|c|}{ Aspectos sociales } \\
\hline Alternativas & \multicolumn{2}{|c|}{ CS8 } & CS9 & CS10 & CS11 & CS12 & CS13 \\
\hline Eólica & \multicolumn{2}{|l|}{3} & 5 & 1 & 4 & 4 & 4 \\
\hline Fotovoltaica & \multicolumn{2}{|l|}{1} & 1 & 1 & 1 & 4 & 4 \\
\hline Undimotriz & 3 & \multicolumn{2}{|c|}{\begin{tabular}{|l|l}
3 & \\
\end{tabular}} & \begin{tabular}{l|l}
5 & \\
\end{tabular} & 4 & 5 & 2 \\
\hline \multicolumn{8}{|c|}{ Aspectos económicos } \\
\hline Alternativas & CE14 & CE15 & CE16 & CE17 & CE18 & CE19 & CE20 \\
\hline Eólica & 4 & 4 & 4 & 4 & 4 & 4 & 2 \\
\hline Fotovoltaica & 4 & 1 & 4 & 5 & 5 & 3 & 5 \\
\hline Undimotriz & 5 & 5 & 2 & 2 & 2 & 5 & 1 \\
\hline
\end{tabular}

Fuente: Elaboración propia.

Aunque este estudio presenta sólo tres alternativas, 20 criterios son utilizados para análisis, abordando los aspectos ambientales, sociales y económicos, que pueden ser relativamente complejos para el reto del modelaje de preferencia. Así, el modelaje fue cuidadosamente llevado a cabo con las informaciones de los expertos en el área de fuentes de energía limpia y decisores.

En el modelaje de preferencia, uno va a ponderar los criterios de acuerdo con las preferencias del decisor, o sea, el decisor debe valorar los criterios y los clasifica de acuerdo con su preferencia (KEENEY; RAIFFA, 1976).

El método multicriterio utilizado para este estudio específicamente debe ser compatible con la racionalidad no compensatoria, o sea, el resultado de un criterio no puede ser equilibrado en otros. No hay trade-off con los criterios del método no compensatorio, un mal desempeño no puede compensar un buen desempeño en otro. La problemática inherente al problema de decisión en este estudio es la ordinación. El método PROMETHEE II fue escogido porque establece un pre-orden completo entre las alternativas. Aunque, las principales ventajas de este método están en la sencillez del resultado ordinado y en la flexibilidad del proceso de elicitación; no hay en el método reglas específicas para la determinación de los pesos de los criterios (CLEMENTE; de ALMEIDA; de ALMEIDA-FILHO, 2016).

El método PROMETHEE presenta seis tipos de funciones: Tipo $1=$ Criterio usual, Tipo 2 = Forma de $\mathrm{U}$, Tipo $3=$ Umbral de preferencia con forma de $\mathrm{V}$, Tipo 4 = Pseudo criterio escalonado, Tipo 5 = Área de indiferencia y Tipo 6 = Gaussiano (BRANS, MARESCHAL, 2005). 
Considerando las seis funciones del método PROMETHEE, el criterio usual fue adoptado. Para este tipo, los valores negativos o cero asumen el valor cero, la función de preferencia va a ser igual a uno. Esto va a aplicarse para los criterios de maximización; para los criterios de minimización el concepto opuesto es utilizado.

Para simplificar el proceso de toma de decisión de este estudio, el software Visual PROMETHEE 1.4 fue utilizado. La herramienta presenta muchas oportunidades de análisis, ofreciendo al decisor la situación más adecuada, más rápida, más sencilla y más eficiente. De acuerdo con Drazic et al. (2016), este software también ofrece el análisis de resultados parciales, con la posibilidad de la solución más adecuada para el problema del decisor.

Después de la definición de los criterios y sus pesos, y, la ordinación de preferencia, se introdujeron los dados en el software visual PROMETHEE, con el propósito de obtener los resultados de salida del PROMETHEE II, utilizando la función usual. Pero, es imprescindible destacar que la clasificación es llevada a cabo de manera que ' +1 ' es considerada la de mayor preferencia y la más leve preferencia es ponderada por ' -1 '. Así, la alternativa que presenta el desempeño ' +1 ', o sea, el valor máximo, es considerada la fuente de energía renovable más viable para la inversión de los puertos brasileños (DRAZIC et al., 2016).

Para la evaluación de los resultados obtenidos en la Figura I, es posible observar que la fuente más viable para implementación en los puertos brasileños es la energía fotovoltaica, que presenta un mayor grado de preferencia. La energía eólica presenta una preferencia positiva, aunque no es superior a la de la fotovoltaica. La energía undimotriz no es considerada viable. Presenta el peor grado de preferencia, con un desempeño negativo.

Figura I. Análisis de los resultados

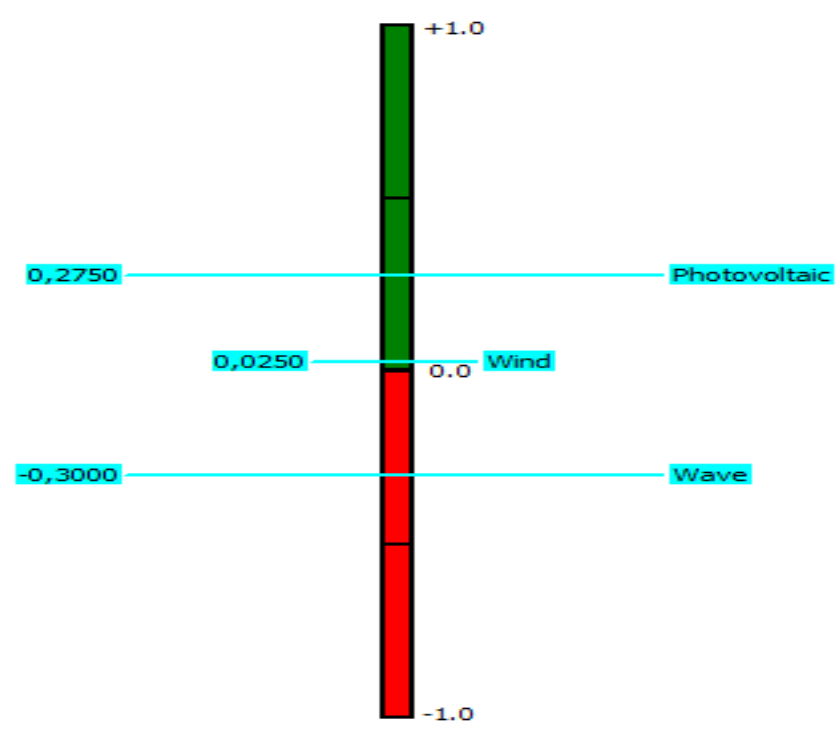

Fuente: Elaboración propia.

Los aspectos de la gestión de sostenibilidad fueron evaluados por separado, con el propósito de verificar cuál de las tres fuentes presenta menor impacto ambiental, social y económico. Los criterios de cada aspecto fueron ordenados de acuerdo con el grado de preferencia del decisor. Para los aspectos ambientales, los resultados obtenidos son presentados en la Figura II. 
Figura II. Análisis de los aspectos ambientales

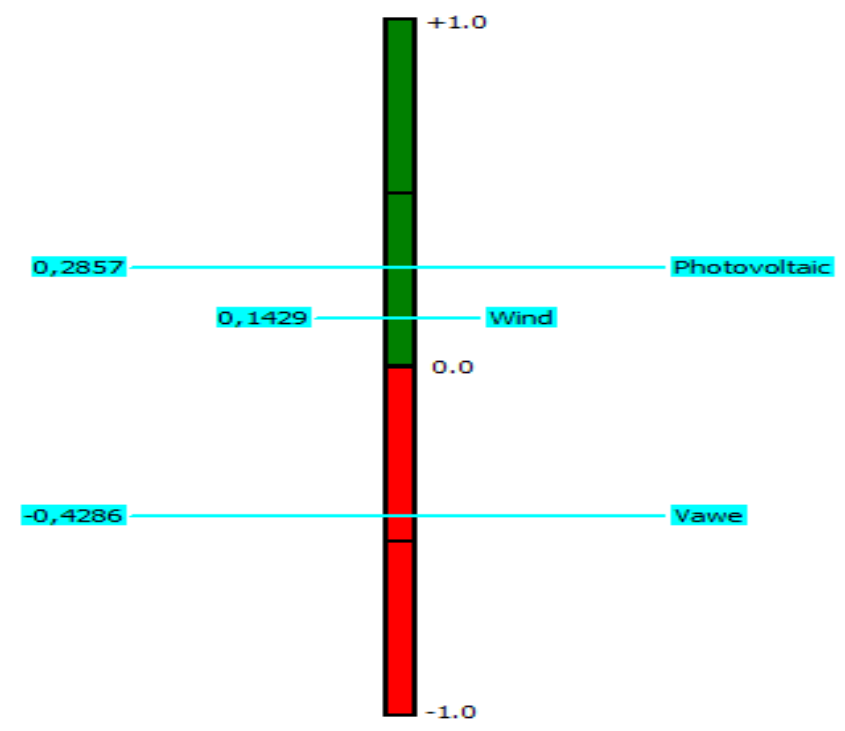

Fuente: Elaboración propia.

En una evaluación detallada de las cuestiones ambientales de las tres fuentes de energía renovable, es posible verificar que el riesgo de contaminación de aguas marinas, la apropiación de extensiones marítimas y la supresión de la vegetación fluvio-marina no son factores de gran impacto para las fuentes de energía fotovoltaica y eólica. Para la energía undimotriz, presentan un riesgo muy elevado, considerado estos criterios.

Cuando uno habla de la necesidad de construir vías de acceso para los emprendimientos de energía renovable, las fuentes de energía eólica y undimotriz tienden a presentar la necesidad de apertura de vías de acceso para los emprendimientos.

Para la energía fotovoltaica, este estudio ha considerado la instalación de placas fotovoltaicas en los techos de los almacenes portuarios; así, no se necesita de apertura de vías de acceso. Igualmente, no hay necesidad de apropiación de extensiones terrestres y supresión de vegetación.

Con respecto a la utilización de productos tóxicos contaminantes en el proceso productivo de los equipos utilizados en los emprendimientos, se puede destacar que palas eólicas usualmente están compuestas de una mixtura de materiales como, por ejemplo, el polímero termofijo, la resina epoxi, reforzado con fibras que, usualmente, son de vidrio. Estos productos son considerados tóxicos y contaminantes (HINDRICH; KLEINBACH, 2004).

La resina está compuesta de productos derivados del petróleo. Así, se está estudiando la viabilidad de la utilización de materiales sostenibles para construir las palas eólicas (HOLMES, 2009).

Segundo Chen et al. (2009), para la energía fotovoltaica, las placas solares son esenciales para la generación de energía fotovoltaica, sin estos no es posible transformar la radiación solar en energía eléctrica. Aunque, sea construidos de materiales semiconductores, o sea, silicio cristalino y arseniato de galio, que son productos considerados extremadamente tóxicos y contaminantes. 
Para la energía undimotriz, los expertos en las fuentes de energía renovable la Empresa de Investigación Energética-aclaran que, para este tipo de energía, hay gran diversidad de tecnologías en desarrollo para distintas maneras de generación.

La utilización de productos tóxicos y contaminantes en el proceso productivo de los equipos está estrechamente relacionada con la modalidad tecnológica utilizada, con mucha o reducida utilización de productos tóxicos.

Para los aspectos sociales de los emprendimientos de energía renovable, una vez más, la energía fotovoltaica se ha presentado con mayor preferencia; las energías eólica y undimotriz presentan un desempeño negativo, como describe la Figura III.

La energía fotovoltaica, así como, la energía eólica, no han presentado ninguna limitación o interferencia en la navegación y la pesca. Para la energía undimotriz se ha presentado un bajo desempeño en este criterio.

Figura III. Análisis de los aspectos sociales

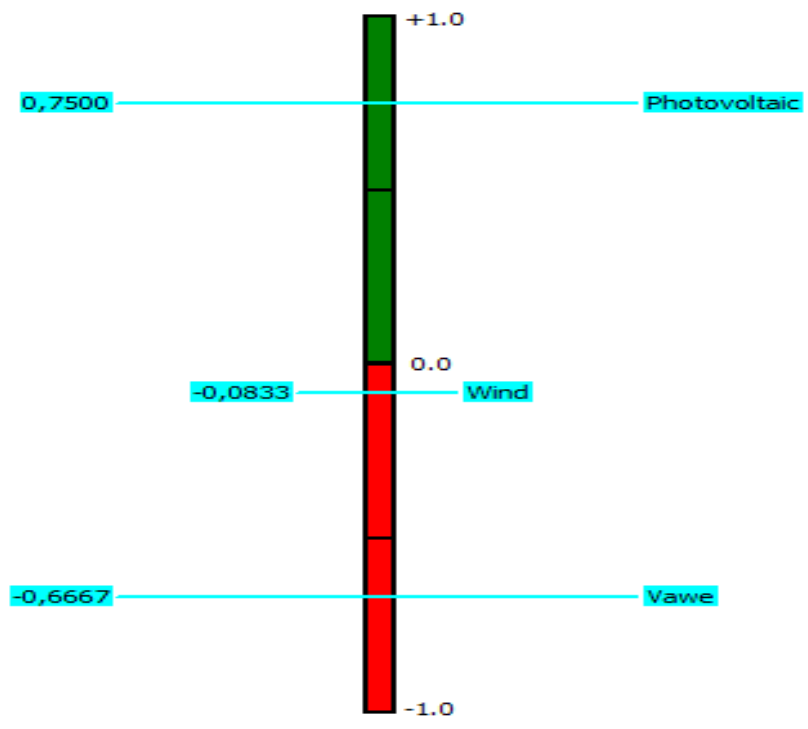

Fuente: Elaboración propia.

Para la evaluación de la generación de ruidos de los emprendimientos, la necesidad de desplazamiento de la comunidad local, la deforestación y la reducción de área verde, es posible destacar que la energía fotovoltaica presenta bajo impacto en estos criterios, en comparación con la energía eólica y undimotriz.

Con relación a los riesgos de salud y seguridad de los trabajadores de un emprendimiento de energía renovable, están sometidos a caídas, daños, riesgos eléctricos, postura incorrecta, espacio confinado, condiciones climáticas, instalaciones en áreas de riesgo, y otras situaciones. Adicionalmente, para la energía undimotriz es necesario verificar la salud y seguridad de las actividades acuáticas. Según los expertos, la energía undimotriz presenta el riesgo más alto en comparación con las otras dos fuentes de energía renovable.

Los aspectos sociales también fueron evaluados. Con respecto al nivel de empleabilidad, los emprendimientos de energía renovable generan un volumen apreciable de empleos directos e indirectos; esto contribuye para el desarrollo económico de la región y el país (IRENA, 2017). La Asociación Brasileña de Energía Solar - ABSOLAR (2016) estima que, para cada megavatio ya instalado de energía solar, son creados 20 o 30 puestos de trabajo, directos e indirectos. En 
comparación, el sector eólico emplea un promedio de 15 personas (directos e indirectos) a cada megavatio instalado. Para la energía unidmotriz, se prevé para 2030 que la fuente de energía empleará un promedio de un millón de trabajadores mundialmente (ESTEBAN; LEARY, 2012).

Finalmente, los aspectos económicos de las fuentes de energía fueron evaluados. Una vez más, la energía fotovoltaica es la más favorable. La energía eólica ha demostrado ser poco favorable y la energía undimotriz es totalmente desfavorable, como presentado en la Figura IV.

Es imprescindible destacar que la energía fotovoltaica no es la fuente que presenta más eficiencia. Adicionalmente, la energía fotovoltaica presenta una alta tasa de depreciación anual, o sea, la vida útil de los equipos es corta, con impacto directo en los resultados de la empresa. La energía undimotriz presenta dos pontos positivos con relación a los aspectos económicos en comparación con las otras dos fuentes de energía. Esta fuente presenta mejor eficiencia y menor tasa de depreciación; para otros criterios, presenta puntos negativos.

Figura VI. Análisis de los aspectos económicos

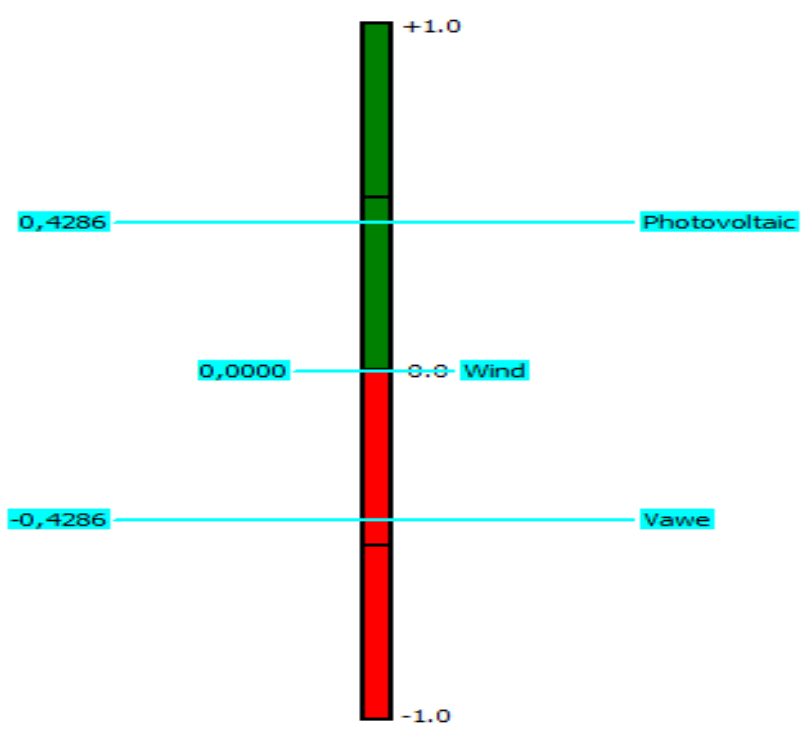

Fuente: Elaboración propia.

Lo que hace atrayente la energía fotovoltaica en los aspectos económicos son los bajos niveles de inversión inicial en comparación con otros emprendimientos. La inversión de la Eletrosul en la ciudad de Florianópolis para transformar su sede administrativa en un complejo de generación fotovoltaica es un ejemplo de esto. La usina fotovoltaica he sido construida encima al edificio administrativo y las plazas de estacionamiento. Se extiende por un área de 8,3 mil metros cuadrados con una inversión de R\$ 9,5 millones de reales (USD 2.33 millones) y capacidad para generar aproximadamente 1.200 megavatios-hora (ELETROSUL, 2018). Así, una inversión de R\$ 7,92 (USD 1.97) por kilovatio-hora.

Para la energía eólica, la Asociación de Ciudades de la Región Montañosa AMURES (2014), ha informado que la inversión inicial en la usina eólica de Bom Jardim da Serra fue de aproximadamente $R \$ 1,258$ milles de millones (USD 310 millones), con una capacidad de producción de 222 megavatios/hora, o sea, un promedio de R\$ 5.670 (USD 1,413) por kilovatio-hora de inversión. 
De acuerdo con el Instituto Alberto Luiz Coimbra de Postgrado e Investigación de Ingeniería - COPPE/UFRJ (2013), se pretende realizar un proyecto para inversión en la energía undimotriz en Rio de Janeiro. Debe ser más eficiente y tener costos más bajos de lo que fue hecho en el Puerto de Pecém; también debe tener equipos más modernos. El proyecto incluye una inversión de R\$ 9 millones (USD 2.24 millones), con capacidad para generar 100 kilovatio-hora, o sea, aproximadamente R\$ 90 mil (USD 22.43 mil) en inversiones por kilovatio-hora (COPPE/UFRJ, 2013).

Otro punto considerado primordial en los aspectos económicos es el plazo de financiación del emprendimiento. Según informaciones presentadas por el Banco Nacional de Desarrollo Económico y Social - BNDES (2017), el plazo máximo de financiación de un proyecto de energía fotovoltaica es de 20 años. Para la energía eólica es de 16 años y para la energía undimotriz es de 10 años. Segundo CastilloCalzadilla y Macarulla (2018), el emprendimiento fotovoltaico es lo más favorable.

Los incentivos fiscales de los emprendimientos de energía renovable también fueron verificados. Para la energía eólica y fotovoltaica hay suspensión, reducción o exención del Impuesto de Productos Industrializados, Impuesto de Importación, Impuesto sobre la Circulación de Mercancías y Servicios, Impuesto sobre las Transacciones Financieras y el Impuesto sobre la Renta de la Persona Jurídica. En el caso de la energía undimotriz, como no es muy conocida en Brasil, no hay legislación específica para los incentivos fiscales. Sin embargo, es posible reconocer que, para este tipo de emprendimiento, hay reducción de Impuesto sobre la Renta de la Persona Jurídica y exención del Impuesto de Importación y del Impuesto sobre la Circulación de Mercancías y Servicios.

Con los resultados obtenidos de las consecuencias de las alternativas de los criterios, fue realizado un análisis de sensibilidad, con valores variables en la escala de criterios y utilizando cuatro funciones del método PROMETHEE: (1) Usual, (2) Forma de U; (3) Umbral de preferencia con forma de V, y, (4) Gaussiano, como presentado en la Figura $\mathrm{V}$.

Figura V. Análisis de sensibilidad

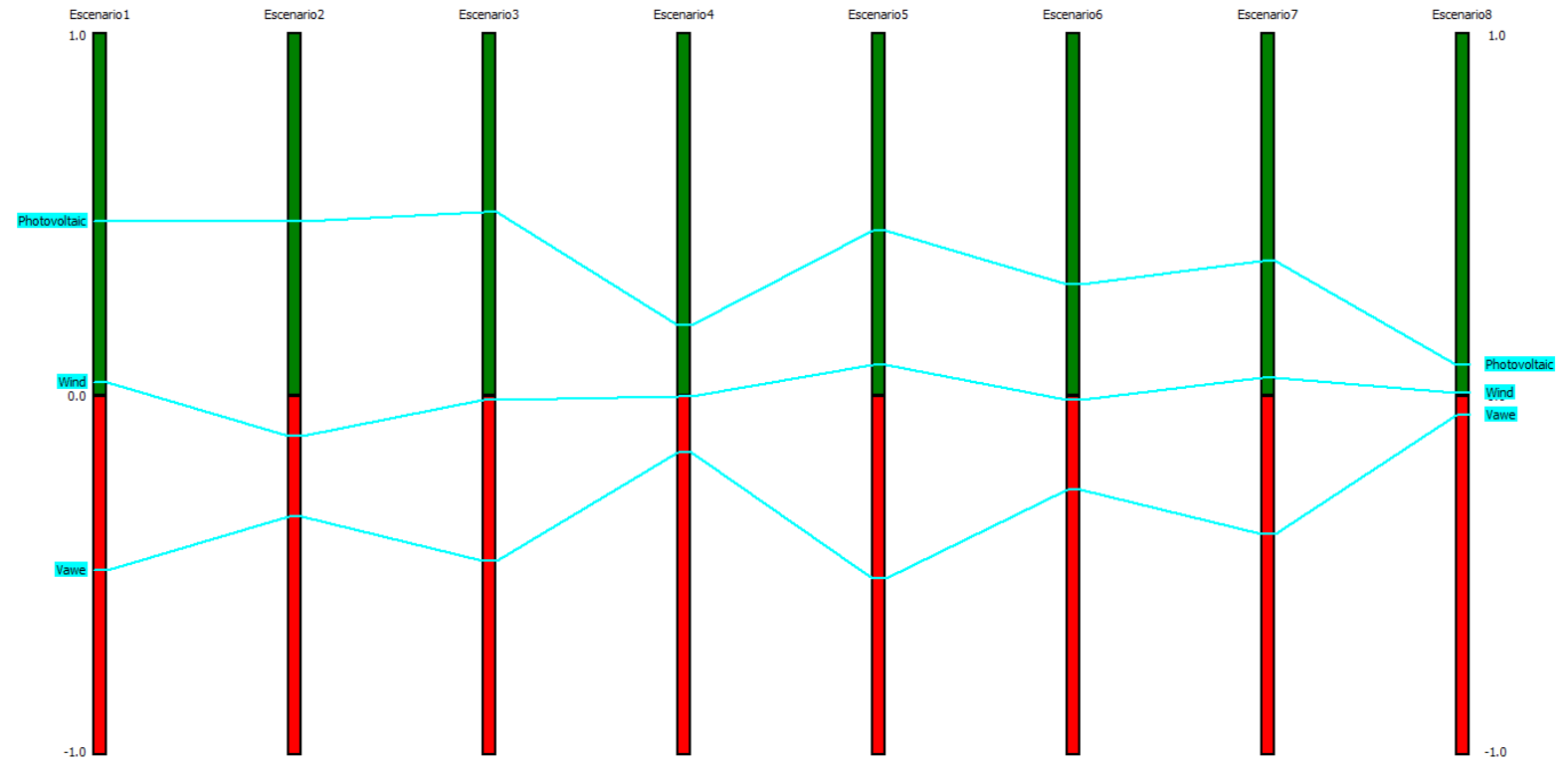

Fuente: Elaboración propia. 
Desde el primero hasta el cuarto escenario, fueron utilizados los mismos pesos y la misma ordenación de los datos originales, pero distintas funciones del método PROMETHEE fueron aplicadas. En el Escenario 1, fue aplicada la función usual, en el Escenario 2 fue aplicada la función Forma de U, en el Escenario 3 fue aplicada la función Umbral de Preferencia con forma de V, y, en el Escenario 4 fue utilizada la función Gaussiana.

Para los otros cuatro escenarios, se ha modificado el valor de los criterios, variando uno o dos puntos para más o para menos, como presentado en la Tabla III.

Tabla III: Análisis de sensibilidad consecuencia de las fuentes de energía renovable

\begin{tabular}{|c|c|c|c|c|c|c|c|}
\hline \multicolumn{8}{|c|}{ Aspectos ambientales } \\
\hline Alternativas & CA1 & CA2 & CA3 & CA4 & CA5 & CA6 & CA7 \\
\hline Eólica & 4 & 2 & 1 & 3 & 2 & 3 & 4 \\
\hline Fotovoltaica & 2 & 2 & 1 & 2 & 3 & 2 & 2 \\
\hline Undimotriz & 3 & 3 & 3 & 3 & 3 & 4 & 3 \\
\hline \multicolumn{8}{|c|}{ Aspectos sociales } \\
\hline Alternativas & CS8 & \multicolumn{2}{|c|}{ CS9 } & \multicolumn{2}{|c|}{ CS11 } & CS12 & CS13 \\
\hline Eólica & 2 & \multicolumn{2}{|c|}{4} & \multicolumn{2}{|c|}{3} & 3 & 3 \\
\hline Fotovoltaica & 1 & \multicolumn{2}{|c|}{1} & \multicolumn{2}{|c|}{1} & 3 & 3 \\
\hline Undimotriz & 3 & \multicolumn{2}{|c|}{3} & \multicolumn{2}{|c|}{3} & 4 & 2 \\
\hline \multicolumn{8}{|c|}{ Aspectos económicos } \\
\hline Alternativas & CE14 & CE15 & CE16 & CE17 & CE18 & CE19 & CE20 \\
\hline Eólica & 4 & 3 & 4 & 4 & 4 & 4 & 4 \\
\hline Fotovoltaica & 3 & 2 & 4 & 4 & 4 & 3 & 4 \\
\hline Undimotriz & 3 & 4 & 3 & 3 & 3 & 3 & 2 \\
\hline
\end{tabular}

Fuente: Elaboración propia.

Después de la variación de las escalas de criterios, distintas funciones del método PROMETHEE fueron aplicadas, o sea, en el Escenario 5, la función usual fue utilizada. En el Escenario 6 la función Forma de U fue aplicada, en el Escenario 7 la función Umbral de Preferencia Forma de V fue aplicada. Y en Escenario 8 la función Gaussiana fue utilizada.

En la Figura V, como se puede percibir, con los nuevos resultados obtenidos, no hubo una modificación significativa, pues la energía fotovoltaica se ha mantenido al tope del ranking. La energía eólica se ha mantenido en la segunda posición y la energía undimotriz está en el último puesto para inversiones en fuentes de energía renovable.

Es importante destacar que, para la implementación efectiva de la energía fotovoltaica, el decisor debe tomar en consideración un modelo, presentando los procedimientos que hay que seguir y evaluar, con respecto a las dimensiones ambientales, sociales y económicos.

Considerando también los indicadores de desempeño para que los procesos de implementación tengan una mejor gestión, $y$, el emprendimiento traiga el resultado deseado por la empresa. 


\section{CONCLUSIÓN}

Los puertos son considerados un sector extremadamente contaminante y, recientemente, se han enfatizado las discusiones con respecto a la gestión de sostenibilidad. Sin embargo, la ANTAQ se ha manifestado en la importancia de la utilización de fuentes de energía renovable con el propósito de hacer los puertos más competitivos en el mercado mundial. Así, el problema de la selección de la fuente de energía renovable más viable para inversión en los puertos brasileños es un tema pertinente para los administradores y para las autoridades portuarias. En este estudio, se ha tratado de combinar el conocimiento presente en las investigaciones científicas, instrucciones normativas, legislación, datos de los emprendimientos ya instalados o en estudio e informaciones de expertos en fuentes de energía renovable para la identificación de la fuente de energía renovable más viable para instalación en los puertos brasileños.

Fueron seleccionados 20 criterios, abordando los aspectos ambientales, sociales y económicos, con el propósito de representar los objetivos seleccionados en este problema específicamente y tres fuentes de energía renovable fueron evaluadas, con respecto a estos criterios. El modelaje de preferencia fue realizado, considerando las preferencias de un gerente administrativo y un coordinador ambiental en un abordaje no compensatoria, a través de la utilización del método PROMETHEE II.

El proceso de elicitación del método PROMETHEE II con los decisores es realizado por medio de un sistema de apoyo a la decisión, por lo cual son ordenados los criterios de acuerdo con la preferencia de los decisores. Así, son identificadas alternativas de mayor o menor preferencia, en el grado de ordenación.

Es importante destacar que la alternativa de mayor preferencia, no es la alternativa de mejor eficiencia, muchas veces considerado el único atributo en el proceso de toma de decisión. Es necesario que un conjunto de criterios sea evaluado para obtener el mejor resultado, cuando se habla de un proceso de selección.

\section{AGRADECIMIENTOS}

Los autores desean agradecer a la Coordinación para el Perfeccionamiento de Personal de Nivel Superior de Brasil (CAPES) por el apoyo financiero parcial a la investigación, la ELETROSUL y la Empresa de Investigación Energética por las contribuciones de los datos proporcionados por expertos. 


\title{
Use of renewable energy sources in brazilian ports
}

\begin{abstract}
This research presents a decision model to solve a selection problem, identifying which renewable energy source is the most viable to invest in Brazilian ports, considering the aspects of environmental, social and economic management, using the PROMETHEE Method (Preference Ranking Organization Method for Enrichment Evaluations). Organizational method for enrichment evaluations) by applying Visual PROMETHEE 1.4 software. However, this research evaluated three sources of clean energy (wind, photovoltaic, and wave), defining twenty criteria, based on existing knowledge in scientific research, regulatory instructions, legislation, data from projects already installed or under study. Noting that these criteria were evaluated by experts from the Energy Research Company (EPE, in Brazilian Portuguese) on a five-point liker scale. However, the evaluation carried out in this study reveals that photovoltaic energy is the most viable renewable energy source for Brazilian ports to invest.
\end{abstract}

KEY WORDS: Renewable energy, multicriteria, ports, sustainability, PROMETHEE. 


\section{REFERÊNCIAS}

ABSOLAR Associação Brasileira de Energia Solar Fotovoltaica (2016) Notícia e eventos. Notícias externas. Solar criará ao menos 60 mil empregos no Brasil. Disponible en: <http://www.absolar.org.br/noticia/noticias-externas/solar-criaraao-menos-60-mil-empregos-no-brasil.html> Acesseso en: 08/01/ 2018.

ACCIARO, M.; GHIARA, H.; CUSANO, M.I. Energy management in seaports: A new role for port authorities. Energy Policy, v. 7, p. 4-12, 2014.

de ALMEIDA, A.T.; CAVALCANTE, C.A.V.; ALENCAR, M.H.; FERREIRA, R.J.P.; de ALMEIDA-FILHO, A.T.; GARCES, T.V. Multicriteria and Multi-objective Models for Risk, Reliability and Maintenance Decision Analysis. International Series in Operations Research \& Management Science. 231, Springer, New York, NY, USA, 2015.

AMURES Associação dos Municípios da Região Serrana (2014) Usina eólica de Bom Gardim da Serra e Agua Doce começam em março. Disponível en: <http://www.amures.org.br/noticias/index/ver/codMapaltem/41771/codNoticia /115909> Acesseso en: 10/01/2018.

BALDENEGRO, C. Photovoltaic solar power at the port of los angeles. 13th Triennial International Conference, p. 188-194, 2013.

BNDES Banco Nacional de Desenvolvimento Econômico e Social (2017) Financiamentos de geração de energia renovável e alternativa. Disponible en: <http://www.bndes.gov.br/wps/portal/site/home/financiamento/produto/bndes -finem-energia> Acesseso en: 26/07/2017.

BRANS, J.P.; VINCKE, P.A. A preference ranking organization method: The PROMETHEE method for MDCM. Management Science, v. 31, p. 647-656, 1985.

BRANS, J.P.; MARESCHAL, B. Multiple criteria decision analysis: State of the art surveys. Springer, 2005.

BRASIL. LEI № 8.630 de 25 de fevereiro 1993. Dispõe sobre o regime jurídico da exploração dos portos organizados e das instalações portuárias (revogada). Disponible en: <http://www.planalto.gov.br/ccivil_03/leis/L8630.htm>. Acesseso en: 30/05/2017.

BRASIL. LEI № 12.815 de 05 de junho de 2013. Dispões sobre a exploração direta e indireta pela união de portos e instalações portuárias sobre as atividades desempenhadas pelos operadores portuários. Disponible en: 
<http://www.planalto.gov.br/ccivil_03/_ato2011-2014/2013/Lei/L12815.htm>. Acesseso en: 23/08/2016.

CASTILLO-CALZADILLA. T.; MACARULLA, A.M.; BORGES, C.E. Design of sizing algorithms for a direct current off-grid photovoltaic intallation. IEEE Latin America Transactions, v. 8, p. 2178-2176, 2018.

ÇELIKLE, D.; ASKAN, Z. The development of an attitude scale to assess the attitudes of high school students towards renewable energy sources. Renewable and Sustainable Energy Reviews, v. 54, p. 1092-1098, 2016.

CHEN, N.; ZHANG, X.; BAI, Y.; ZHANG, H. Environmental Friendly PV Power Plant. Energy Procedia, v. 16, p. 32-37, 2011.

CLEMENTE, T.; de ALMEIDA A.T.; de ALMEIDA-FILHO, A. T. Experiments with Surrogate Weights for PROMETHEE. Promethee Days, Montréal, Canadá, 2016.

COPPER/UFRJ (2013) Rio de Janeiro terá geração de energia pelas ondas do mar. Disponible en: <http://www.coppe.ufrj.br/pt-br/planeta-coppenoticias/noticias/rio-de-janeiro-tera-geracao-de-energia-pelas-ondas-do-mar>. Acesseso en: 03/07/2017.

DRAZIC, J.; DUNJIĆ, D.; MUČENSKI, V.; PEŠKO, I. Multi-criteria analysis of variation solutions for the pipeline route by applying the PROMETHEE method. Tehnički Vjesnik, v. 23, p. 599-610, 2016.

ECKERT, E.R.G.; SPARROW, E.M.; GOLDSTEIN, R.J.; SCOTT, C.J.; PFENDER, E.; PATANKAR, S.V.; RAMSEY, J.W. Heat transfer-a review of 1975 literature. International Journal of Heat and Mass Transfer, v.11, p. 1217-1243, 1976.

ELETROSUL (2018) Megawatt Solar. Usina Fotovoltaica Eletrosul. Disponible en: <http://www.eletrosul.gov.br/ampnbsp/energia-solar-fotovoltaica> Acesseso en: 10/01/2018.

ENSSLIN, L.; ENSSLIN, S.R. Processo de construção de indicadores para a avaliação de desempenho. In: V Ciclo de debates - Avaliação de Políticas Públicas, 2009.

ENSSLIN, L.; MONTIBELLER NETO, G.; NORONHA, S.M. Apoio à Decisão. Florianópolis: Insular, 2001. 
FOSSILE, D.K., GOUVEA DA COSTA, S.E.; PINHEIRO DE LIMA, E. The Evolution of Scientific Production on Ports Energy Efficiency Management. III CIDESPORT Congresso Internacional do Desempenho Portuário - Florianópolis/SC, 2016.

Glendenning, I. Ocean Wave Power. Applied Energy, v. 3, p. 197 -222, 1976.

HINDRICH, R.A.; KLEINBACH, M. Energia e o Meio Ambiente, 3. ed, Editora Thomson, São Paulo, 2004.

HOLMES, J.W.; BRONDSTED, P.; SORENSEN, B.F.; JIANG, Z.; SUN, Z.; CHEN, X. Development of bamboo-based composite as a sustainable green material for wind turbine blades. Wind Engineering, v. 33, p. 197-210, 2009.

IRENA (2017) International Renewable Energy Agency. About IRENA. Work programme. Disponible em <http://www.irena.org/menu/index.aspx?mnu=cat\&PriMenulD=13\&Cat|D=100>. Acesseso en: 07/08/2017.

KEENEY, R.L.; RAIFFA, H. Decision Analysis withMultiple Conflicting Objectives. Wiley \& Sons, New York, NY, USA, 1976.

LIRN, T.; WU, Y.J.; CHEN, Y.J. Green performance criteria for sustainable ports in Asia. International Journal of Physical Distribution and Logistics Management, v. 43, p. $427-451,2013$.

MACHARIS, C.; SPRINGAEL, J.; BRUCKER, K.; VERBEKE, A. Promethee and AHP: the design of operational synergies in multicriteria analysis: strengthening Promethee with ideas of AHP. European Journal of Operational Research, v. 153, p. 307-317, 2004.

MORAES, D.C.; ALENCAR, L.H.; de ALMEIDA, A.T.; CLEMENTE, T.R.N. PROMETHEEROC model for assessing the readiness of technology for generating energy. Mathematical Problems in Engineering, v. 7, p. 1-11, 2015.

MOUTINHO, V.; ROBAINA, M. Is the share of renewable energy sources determining the $\mathrm{CO} 2 \mathrm{kWh}$ and income relation in electricity generation? Renewable and Sustainable Energy Reviews, v. 65, p. 902-914, 2016.

PASKA, J.; SURMA, T. Electricity generation from renewable energy sources in Poland. Renewable Energy, v. 71, p. 286-294, 2014. 
RAMOS, V.; CARVALHO R, ALVAREZ, M.; SÁNCHEZ, M.; IGLESIAS, G. A port towards energy self-sufficiency using tidal stream power. Energy, v. 71, p. 432444, 2014.

ROOS, E.C.; KLIEMANN NETO, F.J. Gestão ambiental portuária considerando os aspectos ambientais e financeiros: uma revisão da literatura das práticas nacionais e internacionais. II CIDESPORT Congresso Internacional do Desempenho Portuário - Florianópolis/SC, 2015.

Recebido: 22 out. 2019

Aprovado: $07 \mathrm{dez} .2019$

DOI: $10.3895 /$ rbpd.v9n1.10988

Como citar: FOSSILE, D. K.; COSTA, S. E. G.; LIMA, E. P. Utilización de fuentes de energías renovables en los puertos brasileños. R. bras. Planej. Desenv. Curitiba, v. 9, n. 1, p. 02-18, jan./abr. 2020. Disponível em: $<$ https://periodicos.utfpr.edu.br/rbpd>. Acesso em: XXX.

Correspondência:

Dayla Karolina Fossile

Rua Imac. Conceição, 1155 - Prado Velho - Curitiba - PR

Direito autoral: Este artigo está licenciado sob os termos da Licença CreativeCommons-Atribuição 4.0 Internacional. 